\title{
Angiogenesis Dysregulation in Psoriatic Arthritis: Molecular Mechanisms
}

\author{
Francesco Paolo Cantatore, ${ }^{1}$ Nicola Maruotti, ${ }^{1}$ \\ Addolorata Corrado, ${ }^{1}$ and Domenico Ribatti ${ }^{2,3}$ \\ ${ }^{1}$ Rheumatology Clinic, Department of Medical and Surgical Sciences, University of Foggia Medical School, Foggia, Italy \\ ${ }^{2}$ Department of Basic Medical Sciences, Neurosciences and Sensory Organs, University of Bari Medical School, Bari, Italy \\ ${ }^{3}$ National Cancer Institute "Giovanni Paolo II", Bari, Italy
}

Correspondence should be addressed to Francesco Paolo Cantatore; francescopaolo.cantatore@unifg.it

Received 1 April 2017; Revised 5 June 2017; Accepted 13 June 2017; Published 19 July 2017

Academic Editor: Ruben Queiro-Silva

Copyright (C) 2017 Francesco Paolo Cantatore et al. This is an open access article distributed under the Creative Commons Attribution License, which permits unrestricted use, distribution, and reproduction in any medium, provided the original work is properly cited.

\begin{abstract}
There is evidence that psoriatic arthritis is closely linked to angiogenesis. Morphological changes described in blood vessels of psoriatic arthritis joints suggest the presence of a dysregulated angiogenesis resulting in the formation of immature vessels. Even if the reason of this inefficient angiogenesis is still unclear, an imbalance between angiogenic and antiangiogenic factors is probably responsible for inducing a dysregulated angiogenesis in psoriatic arthritis, which seems to be involved in its pathogenesis and clinical features. Nevertheless, among chronic arthritides, while angiogenesis in rheumatoid arthritis has been largely studied with a great amount of literature data, limited data on angiogenesis role in psoriatic arthritis are available. This review article is focused on current knowledge on the mechanisms responsible for dysregulated angiogenesis in psoriatic arthritis.
\end{abstract}

\section{Introduction}

Psoriatic arthritis (PsA) is a chronic arthritis, associated with psoriasis, classified with seronegative spondyloarthritis. It is characterized by involvement of metacarpophalangeal and interphalangeal joints of the hands and feet, as well as ankles and knees, often with extra-articular involvement, including eye and/or bowel involvement, sometimes with sacroiliac joints and/or spinal involvement. Joint lesions in PsA are characterized by an erosive arthritis with periosteal reactions occurring in enthesitis and syndesmophytes occurring in spondylitis. In particular, enthesitis and dactylitis are distinctive clinical features of PsA. As demonstrated by ultrasonography and magnetic resonance imaging, enthesis may be the first site involved by inflammation in PsA [1]. Other PsA distinctive features are the absence of serological tests for rheumatoid factor and anti-cyclic citrullinated peptide (CCP) antibodies. PsA is often associated with HLA-B27 in patients with axial involvement [2]. Although the global features of synovitis are not different between rheumatoid arthritis (RA) and PsA, synovium inflammation in PsA is characterized by more intense hypervascularity and infiltration of polymorphonuclear leukocytes [3, 4]. Even if PsA pathogenesis is still unclear, angiogenesis plays a crucial role in the early events in PsA. This review article is focused on the analysis of the current knowledge on the mechanisms responsible for angiogenesis dysregulation occurring in PsA.

\section{Angiogenesis}

Angiogenesis, the formation of new capillaries from preexisting vessels, plays an important role in synovitis pathogenesis. Angiogenesis begins with the production of angiogenic factors which are responsible for the activation of endothelial cells, which, in turn, secrete proteolytic enzymes such as matrix metalloproteinases (MMPs) and plasminogen activators. These enzymes degrade the basement membrane and the perivascular extracellular matrix. Subsequently, endothelial cells proliferate and migrate into the perivascular area. New vessel formation is then completed by lumenation of these "primary sprouts" forming "capillary loops" and by the synthesis of a new basement membrane. The proliferation 
of endothelial cells of these "primary sprouts" and their migration lead to the generation of secondary and further generations of vascular sprouts [5].

The regulation of these events is due to the net balance between angiogenic and antiangiogenic factors [6]. Vascular endothelial growth factor (VEGF) and fibroblast growth factor (FGF) family members, platelet-derived growth factor (PDGF), tumor necrosis factor-alpha (TNF- $\alpha$ ), transforming growth factor-alpha and transforming growth factor-beta (TGF- $\alpha$ and TGF- $\beta$ ), interleukins (ILs), chemokines, angiogenin, and angiopoietins (Angs) are the main angiogenic factors. On the other hand, angiostatin, endostatin, and thrombospondin inhibit angiogenesis [7-9]. An imbalance between these positive and negative factors, with a predominance of angiogenic factors, or downregulation of inhibitory regulators, is involved in impaired angiogenesis which has been observed in several autoimmune diseases $[6,10,11]$.

Angiogenesis appears to be a first-order event in psoriatic arthritis, as in RA. Alterations in the vascular morphology of the nail folds of patients affected by psoriasis without nail disease have been seen by microscopic examination [12], as well as an increase in the number of blood vessels and morphological vascular alterations, such as tortuous and elongated blood vessels, which have been observed in the PsA synovial membrane $[13,14]$.

Morphological vascular alterations in PsA synovial tissue appear to be manifestly distinct from that observed in RA. In fact, PsA is mainly characterized by tortuous, bushy, elongated vessels, whereas RA is prevalently characterized by straight vessels with regular branching [14]. This suggests that angiogenic pathways are different between PsA and RA, just like the pathogenic mechanisms. The morphological chances described in blood vessels of PsA joints are also observed in psoriatic skin lesions and suggest the presence of a dysregulated angiogenesis resulting in immature vessels [15]. Nevertheless, while angiogenesis in RA has been largely studied and a great amount of data is present in the literature $[10,16]$, there are only limited data on the role of angiogenesis in PsA.

\section{The Role of Hypoxia}

The role of hypoxia has been extensively studied in RA, while few data are available in PsA. Hypoxia in the rheumatoid joint has been demonstrated many years ago by direct measurements on synovial fluid samples from RA patients $[17,18]$. Three mechanisms have been suggested to explain hypoxia in RA synovial tissue: (1) the intermittent closing of capillaries for the increased intra-articular pressure due to synovial hyperplasia, synovial fluid effusion, and joint movements within the rigid joint capsule; (2) the high metabolic demand due to the migration and proliferation of inflammatory cells, with an increment of the distance between proliferating cells and nearby blood vessels; (3) the increased expression of angiotensin converting enzyme (ACE) that induces the formation of angiotensin II which is responsible for vasoconstriction and enhancing hypoxia [19]. It is conceivable that the first two mechanisms may also be involved in PsA, while no data are available about ACE expression in PsA.
In PsA, low in vivo oxygen levels have been demonstrated in PsA synovium [20]. Hypoxia is involved in inducing the expression of angiogenic chemokines, MMPs, and hypoxia inducible factor (HIF) [21-25]. Expression of HIF-1 $\alpha$ by macrophages has been observed principally close to the intimal layer but also in the subintimal area in rheumatoid synovium [26]. HIF induces VEGF transcription via hypoxia response element (HRE) interaction in the promoter of VEGF gene and is recognized as a key event in angiogenesis induction [27]. HIF-1 $\alpha$ subunit stability is regulated by oxygen levels through the enzyme prolyl hydroxylase (PHD) [28]. Hypoxia is also responsible for inducing the expression of nuclear factor- $\kappa \mathrm{B}$ (NF- $\kappa \mathrm{B})$ via decreased PHD-dependent hydroxylation of inhibitor of $\kappa \mathrm{B}$ kinase $\beta$ (IKK $\beta$ ) [29].

Hypoxia induces the formation of reactive oxygen species (ROS), via activation of cellular systems, such as the mitochondrial electron transport chain and NADPH oxidase (NOX). ROS are responsible for oxidative damage that modifies the structure of DNA, proteins, and lipids and are involved in angiogenesis, endothelial cell differentiation, proliferation, and migration [30-33]. A significantly increased expression of NOX-2, which is the membranebound catalytic subunit of NOX, has been observed in a study on fifty-four patients with active inflammatory arthritis (33 with RA and 21 with PsA) [34]. High NOX-2 expression was correlated with low synovial $\mathrm{PO}_{2}$ levels and with high expression of VEGF, Ang-2, factor VIII, neural cell adhesion molecule, and $\alpha$-smooth muscle actin. Moreover, a decrease in NOX-2 expression and an increase in in vivo synovial $\mathrm{PO}_{2}$ levels have been found in patients treated with anti-TNF- $\alpha$ [34].

Hypoxia has been correlated to altered bioenergetic and increased metabolic turnover in inflamed joints [35]. In fact, hypoxia is involved in mitochondrial dysfunction and induces a switch to glycolysis, supporting abnormal angiogenesis. Hypoxia and increased glycolytic metabolites are responsible for the expression of HIF- $1 \alpha$ and NF- $\kappa$ B. These transcription factors induce the expression of angiogenic growth factors, inflammatory cytokines, and extracellular membrane components which in turn are involved in further glycolysis [36].

\section{Angiogenic Factors in PSA}

Increased levels of several angiogenic factors, such as VEGF, TGF- $\beta$, PDGF, and Angs, have been found in psoriasis $[37,38]$. Increasing evidence underlines the importance of numerous angiogenic factors also in PsA (Table 1). Angs and VEGF expression has been demonstrated in perivascular areas of PsA synovial membrane and increased expression of VEGF and TGF- $\beta$ has been found in the synovial fluid in early PsA [15, 39]. Ang-2 and VEGF expression in the synovial membrane has been found to be significantly higher in early PsA than in RA. Moreover, significantly higher VEGF and TGF- $\beta$ levels have been seen in the synovial fluid in early PsA compared to RA [40]. MMP-1 and MMP-3 have been described in the sublining and lining layer cells in the synovial tissue in PsA [41]. Moreover, MMP-9 levels have been found to be significantly higher in the synovial fluids of early PsA 
TABle 1: Angiogenic agents involved in PsA.

\begin{tabular}{lc}
\hline \multicolumn{2}{c}{ Angiogenic factors in PsA } \\
\hline VEGF & {$[40,47,61]$} \\
TGF- $\beta$ & {$[40]$} \\
Ang-1 & {$[40]$} \\
Ang-2 (both stimulator and inhibitor) & {$[40,61]$} \\
MMP-9 & {$[42]$} \\
TNF- $\alpha$ & {$[59-62,67]$} \\
\hline
\end{tabular}

VEGF: vascular endothelial growth factor; TGF- $\beta$ : transforming growth factor- $\beta$; Ang: angiopoietin; MMP-9: matrix metalloproteinase-9; TNF- $\alpha$ : tumor necrosis factor- $\alpha$.

than in early RA. In the synovial membrane, MMP-9 levels have also been found to be higher in early PsA than in early RA, but without a significant difference [42].

Synovial vascular morphology appears to be related to angiogenic factors, such as VEGF, Angs, and MMP-9. In PsA, distinct vascular morphology, characterized by tortuous vessels, has been correlated with VEGF levels [40]. Moreover, in PsA lining layer hyperplasia is less evident than in RA, probably due to impaired apoptosis of lining cells and decreased presence of CD68+ macrophage-like synoviocytes [43].

The concomitant expression of these angiogenic molecules in PsA joints plays a key role in angiogenesis induction, as demonstrated by the more intense activation of an important angiogenic signaling pathway, the NOTCH-DLL4, after stimulation of VEGF and Ang-2 in combination compared with either VEGF or Ang-2 alone [44].

Accumulated evidence shows that PDGF has a key role in the recruitment of pericytes to newly formed vessels, where their primary function is to maintain the vessel integrity. In inflammatory arthritis, both immature and mature blood vessels have been found in the synovium. The presence of immature vessels may be responsible for instability of endothelial-pericyte interactions [45].

VEGF and its receptors, VEGFR-1/Flt1 and VEGFR2/KDR, have been demonstrated in PsA synovial tissue, suggesting VEGF's role in inducing angiogenesis and vascular permeability $[39,46]$. In the synovial tissue, VEGF may be derived from endothelial and synovial cells. Synovial VEGF levels may be upregulated by cytokines, such as IL-1 and TNF- $\alpha$, secreted by inflammatory cells and synoviocytes [46]. Increased levels of VEGF have also been observed in serum obtained from PsA patients [47], produced by macrophages, fibroblasts, neutrophils, and platelets [48, 49]. Nevertheless, Przepiera-Będzak et al. [50] have found comparable VEGF, EGF, and FGF-2 serum levels in PsA patients and controls, even if the authors admit the presence of several limitations in their study including a small number of patients and no group of patients without treatment. In the same study, serum VEGF levels correlated with serological and clinical indicators, such as CRP and BASFI (Bath Ankylosing Spondylitis Functional Index), and disease duration [50]. VEGF polymorphisms have been associated with the onset of psoriasis [51, 52]. A low expression of the T allele of VEGF rs3025039, known as
+936 C/T, has been found in PsA patients when compared with controls [53]. This suggests that this polymorphism has a protective role against the development of PsA. It is interesting to underline that the frequency of the $936 \mathrm{~T}$ allele is significantly increased in RA patients, suggesting that different mechanisms are involved in angiogenesis in PsA and RA [54].

Inflammatory cytokines involved in PsA pathogenesis, such as TNF- $\alpha$, also have angiogenic effects. TNF- $\alpha$ is involved in the induction and upregulation of angiogenic agents, such as VEGF [15]. A similar role is also conceivable for IL-1 [15]. In synovial fibroblasts, TNF- $\alpha$, via stimulation of Toll-like receptor-2 (TLR-2) pathway, induces the translocation of NF- $\kappa \mathrm{B}$, which is responsible for inducing the expression of proinflammatory cytokines and MMPs $[46,55]$. IL-17, produced by T-helper-17 via stimulation of IL-23, induces upregulation of proinflammatory cytokines, neutrophils chemiotaxis, endothelial cell migration [46], suggesting a role for IL-17 and IL-23 in angiogenesis in psoriatic arthritis. Oncostatin M (OSM) is a member of the IL-6 family, which has a role in arthritis pathogenesis [56]. Its involvement in angiogenesis has been seen in RA [57]. More recently, a role for OSM has been described in IL-17 regulation [58]. Thus, it is conceivable that OSM may play a role in PsA angiogenesis.

Therapies with TNF- $\alpha$ inhibitors have been associated with reduced levels of VEGF in sera and skin of patients affected by PsA $[59,60]$. Immunohistochemical studies on synovial and psoriatic lesional skin biopsies obtained from PsA patients treated with anti-TNF- $\alpha$ agents have demonstrated changes in numerous factors involved in angiogenesis regulation [61, 62]. Lower levels of VEGF and VEGFR-2 and a reduced expression of stromal cell-derived factor 1 (SDF1)/CXC motif chemokine 12 (CXCL12) and Tie2 have been found in PsA patients treated with anti-TNF- $\alpha$ agents [61]. Tie2 is a specific receptor for Ang-1 and Ang-2. The Angs and Tie-2 are also important regulators of blood vessel growth, maturation, and function. Ang-1 is characterized by angiogenic effects, whereas Ang-2 has generally an opposing action [63]. Ang-2 levels increased after anti-TNF- $\alpha$ therapy [61]. By considering that Ang-2 acts as both a Tie-2 antagonist and agonist [64], it is conceivable that Ang-2 induces angiogenesis in the presence of VEGF, whereas it is involved in vascular regression when VEGF is downregulated [65]. As suggested by Cañete et al. [61], an increase in the Ang2/VEGF ratio after anti-TNF- $\alpha$ treatment, as well as Tie2 reduction, may be responsible for the consistent reduction of synovial neovascularization in PsA. By considering that studies have shown a synergistic effect of TNF- $\alpha$ and Angs in driving inflammation and angiogenesis [66], it is conceivable that this could have a role in partial responders to anti-TNF- $\alpha$ treatment.

Finally, a significant reduction in the expression of MMP9 and adhesion molecules, such as $\alpha \mathrm{v} \beta 3$ integrin E-selectin, intercellular adhesion molecule-1 (ICAM-1), and vascular cell adhesion molecule-1 (VCAM-1), and in the number of blood vessels in dermis and/or synovium has been demonstrated after anti-TNF- $\alpha$ therapy $[62,67]$. 


\section{Concluding Remarks}

Alterations in blood vessels' morphology in joints and psoriatic skin lesions suggest the presence of a dysregulated angiogenesis resulting in immature vessels in PsA [15]. Pathogenic mechanisms of this inefficient angiogenesis in PsA are still unclear. However, an imbalance between angiogenic and antiangiogenic factors is probably involved in inducing a dysregulated angiogenesis in PsA, which seems to play an important role in its pathogenesis and clinical implications. Further studies are needed to explain the role of angiogenesis in the pathogenesis of PsA and to clarify the mechanism responsible for angiogenesis dysregulation.

\section{Conflicts of Interest}

The authors declare that there are no conflicts of interest.

\section{References}

[1] L. I. Sakkas, I. Alexiou, T. Simopoulou, and M. Vlychou, "Enthesitis in psoriatic arthritis," Seminars in Arthritis and Rheumatism, vol. 43, no. 3, pp. 325-334, 2013.

[2] K. Rajesh, D. O. Kataria, and L. H. Brent, "Spondyloarthropathies," American Family Physician, vol. 69, pp. 2853-2860, 2004.

[3] T. Yamamoto, "Psoriatic arthritis: from a dermatological perspective," European Journal of Dermatology, vol. 21, no. 5, pp. 660-666, 2011.

[4] M. G. van de Sande and D. L. Baeten, "Immunopathology of synovitis: from histology to molecular pathways," Rheumatology, vol. 55, no. 4, pp. 599-606, 2016.

[5] W. Risau, "Mechanisms of angiogenesis," Nature, vol. 386, no. 6626, pp. 671-674, 1997.

[6] M. S. Pepper, "Manipulating angiogenesis: from basic science to the bedside," Arteriosclerosis, Thrombosis, and Vascular Biology, vol. 17, no. 4, pp. 605-619, 1997.

[7] K. M. Dameron, O. V. Volpert, M. A. Tainsky, and N. Bouck, "Control of angiogenesis in fibroblasts by p53 regulation of thrombospondin-1," Science, vol. 265, no. 5178, pp. 1582-1584, 1994.

[8] M. S. O’Reilly, L. Holmgren, Y. Shing et al., "Angiostatin: a novel angiogenesis inhibitor that mediates the suppression of metastases by a Lewis lung carcinoma," Cell, vol. 79, no. 2, pp. 315-328, 1994.

[9] M. S. O’Reilly, T. Boehm, Y. Shing et al., "Endostatin: an endogenous inhibitor of angiogenesis and tumor growth," Cell, vol. 88 , no. 2, pp. 277-285, 1997.

[10] N. Maruotti, F. P. Cantatore, E. Crivellato, A. Vacca, and D. Ribatti, "Angiogenesis in rheumatoid arthritis," Histology and Histopathology, vol. 21, no. 4-6, pp. 557-566, 2006.

[11] N. Maruotti, F. P. Cantatore, B. Nico, A. Vacca, and D. Ribatti, "Angiogenesis in vasculitides," Clinical and Experimental Rheumatology, vol. 26, no. 3, pp. 476-483, 2008.

[12] M. Bhushan, T. Moore, A. L. Herrick, and C. E. M. Griffiths, "Nailfold video capillaroscopy in psoriasis," British Journal of Dermatology, vol. 142, no. 6, pp. 1171-1176, 2000.

[13] D. Veale, G. Yanni, S. Rogers, L. Barnes, B. Bresnihan, and O. Fitzgerald, "Reduced synovial membrane macrophage numbers, elam-1 expression, and lining layer hyperplasia in psoriatic arthritis as compared with rheumatoid arthritis," Arthritis \& Rheumatism, vol. 36, no. 7, pp. 893-900, 1993.
[14] R. J. Reece, J. D. Canete, W. J. Parsons, P. Emery, and D. J. Veale, "Distinct vascular patterns of early synovitis in psoriatic, reactive, and rheumatoid arthritis," Arthritis and Rheumatism, vol. 42, no. 7, pp. 1481-1484, 1999.

[15] D. J. Veale, C. Ritchlin, and O. FitzGerald, "Immunopathology of psoriasis and psoriatic arthritis," Annals of the Rheumatic Diseases, vol. 64, supplement 2, pp. 26-29, 2005.

[16] H. A. Elshabrawy, Z. Chen, M. V. Volin, S. Ravella, S. Virupannavar, and S. Shahrara, "The pathogenic role of angiogenesis in rheumatoid arthritis," Angiogenesis, vol. 18, no. 4, pp. 433-448, 2015.

[17] K. H. Falchuk, E. J. Goetzl, and J. P. Kulka, "Respiratory gases of synovial fluids: an approach to synovial tissue circulatorymetabolic imbalance in rheumatoid arthritis," The American Journal of Medicine, vol. 49, no. 2, pp. 223-231, 1970.

[18] K. Lund-Olesen, "Oxygen tension in synovial fluids," Arthritis and Rheumatism, vol. 13, no. 6, pp. 769-776, 1970.

[19] C. M. Quiñonez-Flores, S. A. González-Chávez, and C. Pacheco-Tena, "Hypoxia and its implications in rheumatoid arthritis," Journal of Biomedical Science, vol. 23, no. 1, article 62, 2016.

[20] C. T. Ng, M. Biniecka, A. Kennedy et al., "Synovial tissue hypoxia and inflammation in vivo," Annals of the Rheumatic Diseases, vol. 69, no. 7, pp. 1389-1395, 2010.

[21] B. Sivakumar, M. A. Akhavani, C. P. Winlove, P. C. Taylor, E. M. Paleolog, and N. Kang, "Synovial hypoxia as a cause of tendon rupture in rheumatoid arthritis," Journal of Hand Surgery, vol. 33, no. 1, pp. 49-58, 2008.

[22] M. A. Akhavani, L. Madden, I. Buysschaert, B. Sivakumar, N. Kang, and E. M. Paleolog, "Hypoxia upregulates angiogenesis and synovial cell migration in rheumatoid arthritis," Arthritis Research and Therapy, vol. 11, no. 3, article R64, 2009.

[23] B. Muz, M. N. Khan, S. Kiriakidis, and E. M. Paleolog, "Hypoxia: the role of hypoxia and HIF-dependent signalling events in rheumatoid arthritis," Arthritis Research and Therapy, vol. 11, article 201, 2009.

[24] S. Konisti, S. Kiriakidis, and E. M. Paleolog, "Hypoxia-a key regulator of angiogenesis and inflammation in rheumatoid arthritis," Nature Reviews Rheumatology, vol. 8, no. 3, pp. 153162, 2012.

[25] A. Marrelli, P. Cipriani, V. Liakouli et al., "Angiogenesis in rheumatoid arthritis: a disease specific process or a common response to chronic inflammation?" Autoimmunity Reviews, vol. 10, no. 10, pp. 595-598, 2011.

[26] A. P. Hollander, K. P. Corke, A. J. Freemont, and C. E. Lewis, "Expression of hypoxia-inducible factor $1 \alpha$ by macrophages in the rheumatoid synovium: implications for targeting of therapeutic genes to the inflamed joint," Arthritis and Rheumatism, vol. 44, no. 7, pp. 1540-1544, 2001.

[27] E. Tischer, R. Mitchell, T. Hartman et al., “The Human gene for vascular endothelial growth factor: multiple protein forms are encoded through alternative exon splicing," Journal of Biological Chemistry, vol. 266, no. 18, pp. 11946-11954, 1991.

[28] A. Palazon, A. Goldrath, V. Nizet, and R. Johnson, "HIF transcription factors, inflammation, and immunity," Immunity, vol. 41, no. 4, pp. 518-528, 2014.

[29] K. M. Oliver, C. T. Taylor, and E. P. Cummins, "Hypoxia. Regulation of $\mathrm{NF} \kappa \mathrm{B}$ signalling during inflammation: the role of hydroxylases," Arthritis Research \& Therapy, vol. 11, article 215, 2009. 
[30] M. Valko, M. Izakovic, M. Mazur, C. J. Rhodes, and J. Telser, "Role of oxygen radicals in DNA damage and cancer incidence," Molecular and Cellular Biochemistry, vol. 266, no. 1-2, pp. 37-56, 2004.

[31] R. Ray and A. M. Shah, "NADPH oxidase and endothelial cell function," Clinical Science, vol. 109, no. 3, pp. 217-226, 2005.

[32] R. S. Frey, M. Ushio-Fukai, and A. B. Malik, "NADPH oxidasedependent signaling in endothelial cells: role in physiology and pathophysiology," Antioxidants \& Redox Signaling, vol. 11, no. 4, pp. 791-810, 2009.

[33] A. Manea, "NADPH oxidase-derived reactive oxygen species: involvement in vascular physiology and pathology," Cell and Tissue Research, vol. 342, no. 3, pp. 325-339, 2010.

[34] M. Biniecka, M. Connolly, W. Gao et al., "Redox-mediated angiogenesis in the hypoxic joint of inflammatory arthritis," Arthritis and Rheumatology, vol. 66, no. 12, pp. 3300-3310, 2014.

[35] R. Garcia-Carbonell, A. S. Divakaruni, A. Lodi et al., "Critical role of glucose metabolism in rheumatoid arthritis fibroblastlike synoviocytes," Arthritis and Rheumatology, vol. 68, no. 7, pp. 1614-1626, 2016.

[36] M. Biniecka, M. Canavan, T. McGarry et al., "Dysregulated bioenergetics: a key regulator of joint inflammation," Annals of the Rheumatic Diseases, vol. 75, no. 12, pp. 2192-2200, 2016.

[37] D. Creamer, R. Jaggar, M. Allen, R. Bicknell, and J. Barker, "Overexpression of the angiogenic factor platelet-derived endothelial cell growth factor/thymidine phosphorylase in psoriatic epidermis," British Journal of Dermatology, vol. 137, no. 6, pp. 851-855, 1997.

[38] K. Kuroda, A. Sapadin, T. Shoji, R. Fleischmajer, and M. Lebwohl, "Altered expression of angiopoietins and Tie2 endothelium receptor in psoriasis," Journal of Investigative Dermatology, vol. 116, no. 5, pp. 713-720, 2001.

[39] U. Fearon, R. Reece, J. Smith, P. Emery, and D. J. Veale, "Synovial cytokine and growth factor regulation of MMPs/TIMPs: implications for erosions and angiogenesis in early rheumatoid and psoriatic arthritis patients," Annals of the New York Academy of Sciences, vol. 878, pp. 619-621, 1999.

[40] U. Fearon, K. Griosios, A. Fraser et al., "Angiopoietins, growth factors, and vascular morphology in early arthritis," The Journal of Rheumatology, vol. 30, no. 2, pp. 260-268, 2003.

[41] D. Kane, L. E. Jensen, S. Grehan, A. S. Whitehead, B. Bresnihan, and O. Fitzgerald, "Quantitation of metalloproteinase gene expression in rheumatoid and psoriatic arthritis synovial tissue distal and proximal to the cartilage-pannus junction," The Journal of Rheumatology, vol. 31, no. 7, pp. 1274-1280, 2004.

[42] A. Fraser, U. Fearon, R. Reece, P. Emery, and D. J. Veale, "Matrix metalloproteinase 9, apoptosis, and vascular morphology in early arthritis," Arthritis and Rheumatism, vol. 44, no. 9, pp. 2024-2028, 2001.

[43] E. Kruithof, D. Baeten, L. De Rycke et al., "Synovial histopathology of psoriatic arthritis, both oligo- and polyarticular, resembles spondyloarthropathy more than it does rheumatoid arthritis," Arthritis Research \& Therapy, vol. 7, no. 3, pp. R569-580, 2005.

[44] W. Gao, C. Sweeney, C. Walsh et al., "Notch signalling pathways mediate synovial angiogenesis in response to vascular endothelial growth factor and angiopoietin 2," Annals of the Rheumatic Diseases, vol. 72, no. 6, pp. 1080-1088, 2013.

[45] A. Kennedy, C. T. Ng, M. Biniecka et al., "Angiogenesis and blood vessel stability in inflammatory arthritis," Arthritis and Rheumatism, vol. 62, no. 3, pp. 711-721, 2010.
[46] T. Yamamoto, "Angiogenic and inflammatory properties of psoriatic arthritis," ISRN Dermatology, vol. 2013, Article ID 630620, 7 pages, 2013.

[47] S. Ballara, P. C. Taylor, P. Reusch et al., "Raised serum vascular endothelial growth factor levels are associated with destructive change in inflammatory arthritis," Arthritis and Rheumatism, vol. 44, no. 9, pp. 2055-2064, 2001.

[48] S. C. Ballara, J. M. Miotla, and E. M. Paleolog, "New vessels, new approaches: angiogenesis as a therapeutic target in musculoskeletal disorders," International Journal of Experimental Pathology, vol. 80, no. 5, pp. 235-250, 1999.

[49] D. A. Walsh, "Angiogenesis and arthritis," Rheumatology, vol. 38, no. 2, pp. 103-112, 1999.

[50] H. Przepiera-Będzak, K. Fischer, and M. Brzosko, "Serum levels of angiogenic cytokines in psoriatic arthritis and SAPHO syndrome," Polish Archives of Internal Medicine, vol. 123, no. 6, pp. 297-302, 2013.

[51] H. S. Young, A. M. Summers, M. Bhushan, P. E. C. Brenchley, and C. E. M. Griffiths, "Single-nucleotide polymorphisms of vascular endothelial growth factor in psoriasis of early onset," The Journal of Investigative Dermatology, vol. 122, no. 1, pp. 209215, 2004.

[52] S. Barile, E. Medda, L. Nisticò et al., "Vascular endothelial growth factor gene polymorphisms increase the risk to develop psoriasis," Experimental Dermatology, vol. 15, no. 5, pp. 368-376, 2006.

[53] C. Butt, S. Lim, C. Greenwood, and P. Rahman, "VEGF, FGF1, FGF2 and EGF gene polymorphisms and psoriatic arthritis," BMC Musculoskeletal Disorders, vol. 8, article 1, 2007.

[54] S. W. Han, G. W. Kim, J. S. Seo et al., "VEGF gene polymorphisms and susceptibility to rheumatoid arthritis," Rheumatology, vol. 43, no. 9, pp. 1173-1177, 2004.

[55] R. Seibl, T. Birchler, S. Loeliger et al., "Expression and regulation of Toll-like receptor 2 in rheumatoid arthritis synovium," American Journal of Pathology, vol. 162, no. 4, pp. 1221-1227, 2003.

[56] S.-H. Kok, C.-Y. Hong, M. Y.-P. Kuo et al., "Oncostatin Minduced CCL2 transcription in osteoblastic cells is mediated by multiple levels of STAT-1 and STAT-3 signaling: an implication for the pathogenesis of arthritis," Arthritis and Rheumatism, vol. 60 , no. 5, pp. 1451-1462, 2009.

[57] U. Fearon, R. Mullan, T. Markham et al., "Oncostatin M induces angiogenesis and cartilage degradation in rheumatoid arthritis synovial tissue and human cartilage cocultures," Arthritis and Rheumatism, vol. 54, no. 10, pp. 3152-3162, 2006.

[58] H. Son, S. H. Lee, S. Lee et al., "Oncostatin M suppresses activation of IL-17/Th17 via SOCS3 regulation in CD4," The Journal of Immunology, vol. 198, no. 4, pp. 1484-1491, 2017.

[59] A. Mastroianni, E. Minutilli, A. Mussi et al., "Cytokine profiles during infliximab monotherapy in psoriatic arthritis," British Journal of Dermatology, vol. 153, no. 3, pp. 531-536, 2005.

[60] P. Cordiali-Fei, E. Trento, G. D'Agosto et al., "Effective therapy with anti-TNF- $\alpha$ in patients with psoriatic arthritis is associated with decreased levels of metalloproteinases and angiogenic cytokines in the sera and skin lesions," Annals of the New York Academy of Sciences, vol. 1110, pp. 578-589, 2007.

[61] J. D. Cañete, J. L. Pablos, R. Sanmartí et al., "Antiangiogenic effects of anti-tumor necrosis factor $\alpha$ therapy with infliximab in psoriatic arthritis," Arthritis and Rheumatism, vol. 50, no. 5, pp. 1636-1641, 2004. 
[62] A. Y. Goedkoop, M. C. Kraan, D. I. Picavet et al., "Deactivation of endothelium and reduction in angiogenesis in psoriatic skin and synovium by low dose infliximab therapy in combination with stable methotrexate therapy: a prospective single-centre study," Arthritis Research \& Therapy, vol. 6, no. 4, pp. R326-334, 2004.

[63] A. Moss, “The angiopoietin: TIe 2 interaction: a potential target for future therapies in human vascular disease," Cytokine and Growth Factor Reviews, vol. 24, no. 6, pp. 579-592, 2013.

[64] S. Tanaka, M. Mori, Y. Sakamoto, M. Makuuchi, K. Sugimachi, and J. R. Wands, "Biologic significance of angiopoietin2 expression in human hepatocellular carcinoma," Journal of Clinical Investigation, vol. 103, no. 3, pp. 341-345, 1999.

[65] I. B. Lobov, P. C. Brooks, and R. A. Lang, "Angiopoietin-2 displays VEGF-dependent modulation of capillary structure and endothelial cell survival in vivo," Proceedings of the National Academy of Sciences of the United States of America, vol. 99, no. 17, pp. 11205-11210, 2002.

[66] S. García, S. Krausz, C. A. Ambarus et al., "Tie2 signaling cooperates with TNF to promote the pro-inflammatory activation of human macrophages independently of macrophage functional phenotype," PLoS ONE, vol. 9, no. 1, Article ID e82088, 2014.

[67] P. Cordiali-Fei, E. Trento, G. D’Agosto et al., "Decreased levels of metalloproteinase- 9 and angiogenic factors in skin lesions of patients with psoriatic arthritis after therapy with anti-TNF- $\alpha$," Journal of Autoimmune Diseases, vol. 3, article 5, 2006. 


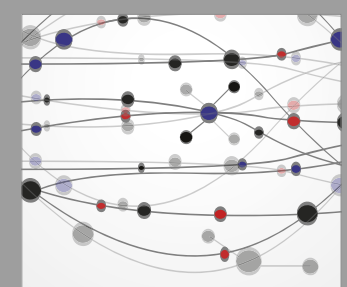

The Scientific World Journal
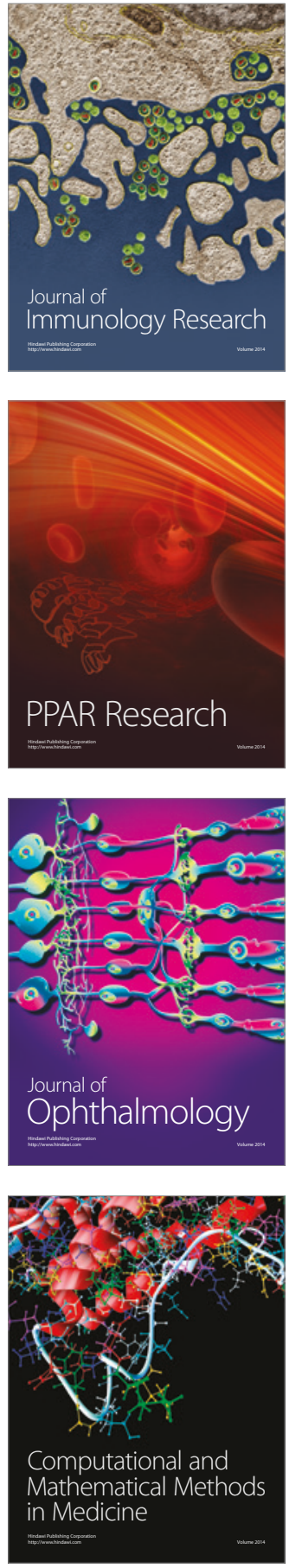

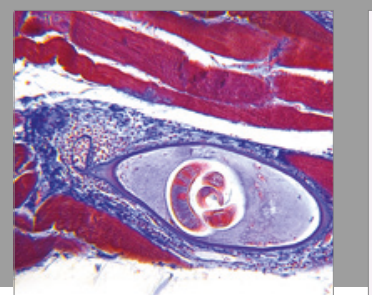

Gastroenterology Research and Practice
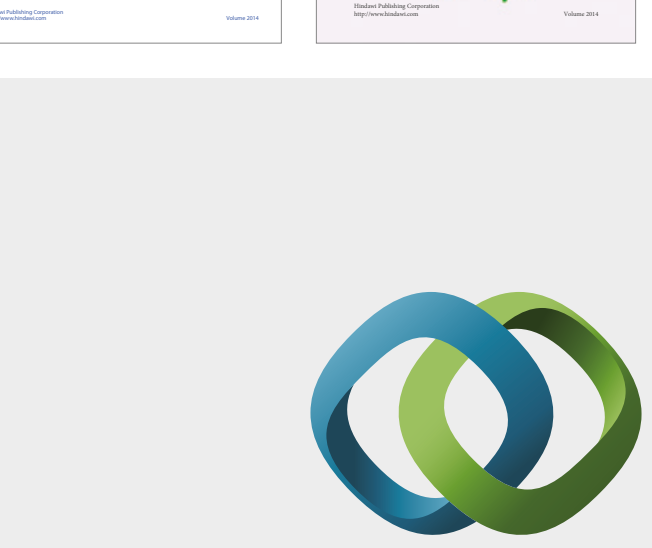

\section{Hindawi}

Submit your manuscripts at

https://www.hindawi.com
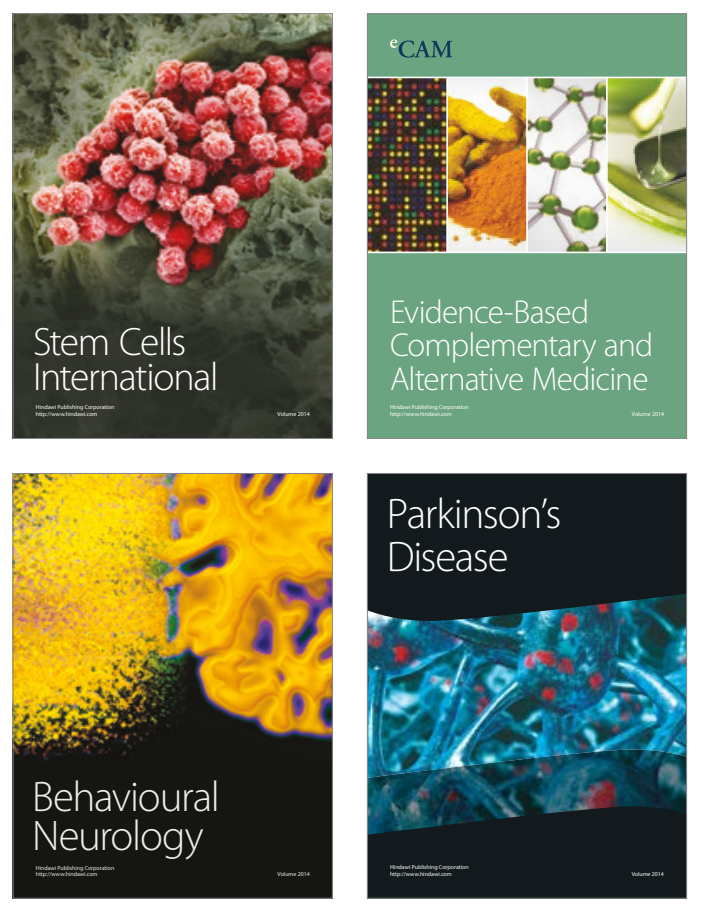
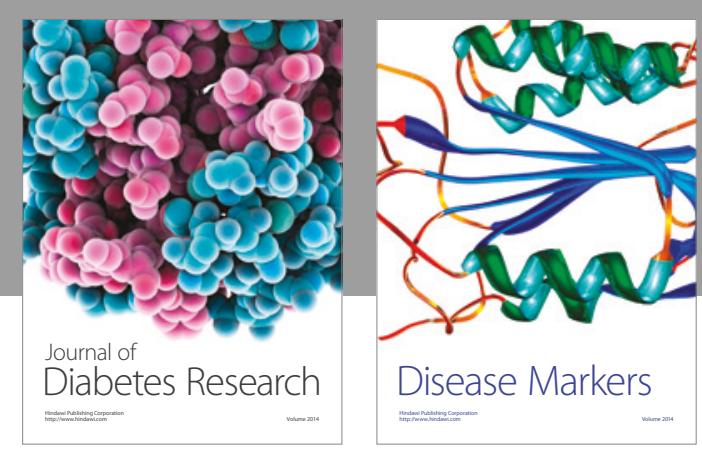

Disease Markers
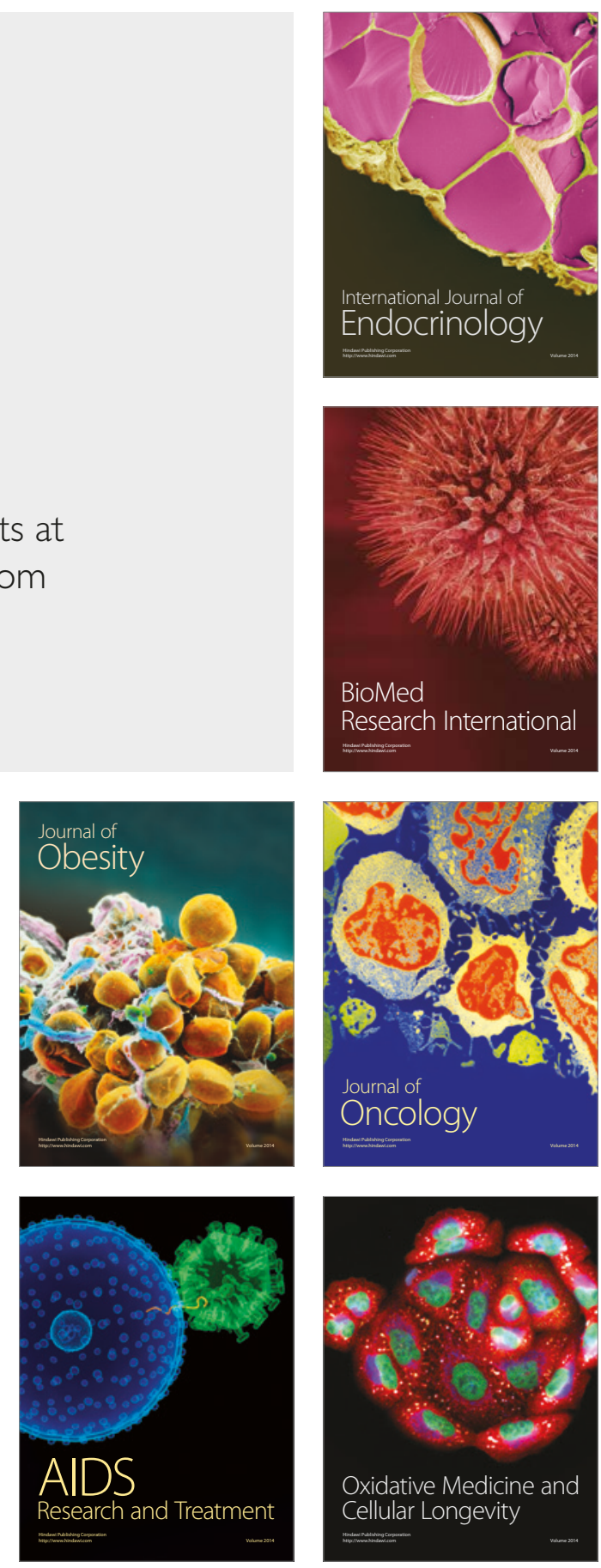